Aus dem pharmazeutisch-chemischen und dem pharmakologischen Institut in Marburg.

\title{
Ueber die Einwirkung von Oidium lactis und Vibrio cholerae auf Cholinchlorid.
}

\author{
Von Dr. A. Ruckert, \\ z. Zt. I. Assistent der Nervenklinik in Halle a. S.
}

(Eingegangen den 20. IX. 1908.)

B ri e g e $\mathbf{r}^{1}$ ) hat zuerst die Vermutung ausgesprochen, daB gewisse Bakterien befähigt werden, aus Cholin durch Abspaltung von einem Molekül Wasser das giftige Neurin zu bilden. So weit ich die Literatur übersehe, hat $\mathrm{Br}$ i e $\mathrm{g} \mathrm{e}^{2}$ ) selbst keine weiteren Experimente unternommen, um dieses interessante Problem weiter zu verfolgen. Dagegen hat $\mathrm{E}$. $\mathrm{Sch} \mathrm{mid} \mathrm{t}^{3}$ ) sechs Versuche angestellt, in denen er zum Teil wässerige Lösung von Cholinchlorid, zum Teil Gelatine mit Cholinzusatz verwandte, die er in den meisten Fällen mit Heuinfus, und in einem Fall mit einer Reinkultur von Bacillus subtilis impfte. Die Wachstumszeit bei seinen Versuchen schwankte zwischen 10 und 14 Tagen, die Temperatur, denen die Kulturen ausgesetzt waren, zwischen 15 und $30^{\circ} \mathrm{C}$. Während vier seiner Versuche, darunter auch bemerkenswerterweise jener mit einer Reinkultur angestellter, negativ waren, war E. $\mathrm{S} \mathrm{ch} \mathrm{mid} \mathrm{t} \mathrm{in} \mathrm{den} \mathrm{ersten} \mathrm{beiden} \mathrm{seiner} \mathrm{Versuche} \mathrm{mit} \mathrm{Heuinfus,}$ also einem Gemisch der verschiedensten aëroben und anaëroben Bakterien glücklicher, insofern er bei der chemischen Rückgewinnung ein Platindoppelsalz gewann, das in seinen Eigenschaften (Farbe, Löslichkeit, Krystallform und Schmelzpunkt: $210-212^{\circ} \mathrm{C}$.) auf die Anwesenheit von Neurin schließen ließ. Die physiologische Untersuchung dieses neurinähnlichen Produktes ergab am freigelegten Froschherz eine ausgesprochene Muskarinwirkung (Verlangsamung der Pulse mit stark verlängerter Diastole).

Auf Veranlassung von Herrn Geheimrat E. S ch mid t in Marburg habe ich diese Versuche in seinem Institute weiter verfolgt und zwar lediglich mit Reinkultur. Ich habe dazu einmal

1) Die Ptomaine, Berlin 1888.

2) Das Cholin als Ptomainbildner, Zeitschr. f. klin. Medizin. Bd. X.

3) Dieses Archiv 1891, 481. 
als Vertreter der gewöhnlichsten Schimmelpilze Oidium lactis und zweitens als pathogenen Mikroorganismus den Vibrio cholerae verwandt. Von dem Cholerastamm erfuhr ich, daß er vor kurzer Zeit in Aegypten isoliert worden war und als höchst virulent angesehen werden durfte ${ }^{1}$ ).

Für die chemische Verarbeitung der Kulturen wäre es wünschenswert gewesen, wenn man dazu einfach wässerige Lösung von Cholinchlorid ohne Zusatz von Nährsubstanzen hätte verwenden können. In längeren Vorversuchen war ich bemüht, reine Cholinchloridlösungen in den verschiedensten Konzentrationen als Nährsubstrat zu verwenden, jedoch wollte es nicht gelingen, selbst nach Zusatz der verschiedensten anorganischen Salze, analog dem Vorgehen $\mathrm{Us} \mathrm{ch}$ ins $\mathrm{k} \mathrm{y} \mathrm{s}^{2}$ ), die Bakterien bezw. Schimmelpilze zum Wachstum zu bringen. Ieh habe deshalb schließlich für Oidium lactis saure Fleischbouillon, für die Choleravibrionen 10\% Peptonlösung verwandt, denen das Cholinhydrochlorid in einer Menge von $0,5 \%$ zugesetzt wurde.

Das Cholinchlorid $\mathrm{C}_{5} \mathrm{H}_{14} \mathrm{~N}$.O.Cl habe ich mir nach $\mathrm{Wurtz}$ synthetisch dargestellt, indem ich äquivalente Mengen von $33 \%$ iger alkoholischer Trimethylaminlösung und von Aethylenchlorhydrin ca. 4-6 Stunden in einem Volh a rd'schen Rohr in einer Wasserbadkanone erhitzte und dann zur Krystallisation beiseite stellte. Nach dem Erkalten war die Flüssigkeit gewöhnlich in toto zu langen Nadeln erstarrt. Nach einmaligem Umkrystallisieren wurden die Krystalle abgesaugt und gesammelt. Die Mutterlauge wurde durch Ueberschichten mit wasserfreiem Aether zu weiterer Krystallisation angeregt und die letzten Mutterlaugen durch Fällung mit konzentrierter alkoholischer Quecksilberchloridlösung im Ueberschuß von dem gebildeten Cholin befreit. Auf diese Weise erbielt. ich in der Regel eine Ausbeute von 50-60\% der theoretisch berechneten Menge. Da das Cholinchlorid bekanntermaßen sehr hygroskopisch ist, wurde es in einem Exsikkator über gebranntem Kalk aufbewahrt. Zur Identifikation des gewonnenen Produktes wurden geringe Mengen $(1,0 \mathrm{~g})$ in $\mathrm{HCl}$-haltigem Wasser gelöst und mit Platinchlorid versetzt. Im Exsikkator über $\mathrm{H}_{2} \mathrm{SO}_{4}$ krystallisierten neben spärlichen, goldgelben, kleinen Oktaedern rotgelbe monokline Tafeln aus. Jene dekrepitierten auf dem Platinblech in trockenem Zustande, ohne einen Rückstand zu hinterlassen, es dürfte sich danach um Platinsalmiak gehandelt haben. Die

1) Die Cholerakultur verdanke ich der Liebenswürdigkeit des Herrn Professor B o $\mathrm{nh}$ of $\mathrm{f}$ in Marburg.

") Us c hin s k y, Centralbl. f. Bakteriologie, Bd. 14, 1893. 
tafelförmigen Krystalle wurden umkrystallisiert, unter der Luftpumpe abgesogen, zwischen Fließpapier getrocknet und gepulvert. Die gepulverte Masse blieb ca. 12-24 Stunden lang über $\mathrm{H}_{2} \mathrm{SO}_{4}$ im Exsikkator stehen, wie ich das bei allen folgenden Schmelzpunktbestimmungen getan habe!

Die Schmelzpunktbestimmung wurde in einem Schwefelsäureapparat ausgeführt und ergab als Mittelwert von drei Bestimmungen $234^{\circ} \mathrm{C}$, , bei welcher Temperatur die Substanz unter Aufschäumen verkohlte. Der Rest dieses Platinsalzes wurde zu einer Elementaranalyse und einer Platinbestimmung verwandt. Die erstere wurde mit Bleichromat und vorgelegter Silberspirale ausgeführt.

1. 0,1925 g Substanz lieferten $0,1352 \mathrm{~g} \mathrm{CO}_{2}$ und $0,0736 \mathrm{~g} \mathrm{H}_{2} \mathrm{O}$.

2. $0,2040 \mathrm{~g}$ Substanz lieferten $0,064 \mathrm{~g}$ Pt.

Gefunden:

\begin{tabular}{|c|c|c|c|}
\hline \multirow{2}{*}{\multicolumn{3}{|c|}{ Gefunden: }} & \multirow{2}{*}{ Berechnet für $\left(\mathrm{C}_{5} \mathrm{H}_{14} \mathrm{NOCl}\right)_{2} \mathrm{PtCl}_{4}$ : } \\
\hline & & 2. & \\
\hline $\mathrm{C}$ & $20,06 \%$ & - & $19,82 \%$ \\
\hline $\mathrm{H}$ & $4,86 \%$ & - & $4,55 \%$ \\
\hline & - & $31,66 \%$ & $31,65 \%$ \\
\hline
\end{tabular}

Nach diesen Analysen konnte es wohl kaum zweifelhaft sein, daß das gebildete Produkt reines Cholinplatinchlorid war.

Da die Kulturen zur Sterilisation an drei aufeinanderfolgenden Tagen je 15 Minuten erhitzt werden mußten, so war zunächst zu entscheiden, wie das Cholinchlorid sich bei dieser Prozedur verhält, bezw. ob es sich dabei zersetzt. $\mathrm{H}$ a $\mathrm{rna} \mathrm{ck}^{\mathbf{1}}$ ) und später $\mathrm{Gr} \mathrm{r} \mathrm{a}^{2}$ ) haben die Ansicht ausgesprochen, daß das Cholin ziemlich leicht in die Vinylbase übergeht. Indes haben E. Sch midt und B ri e g e r unabhängig voneinander nachweisen können, daß die Platinverbindung des Cholins durch 5-6 stündiges Erhitzen auf dem Wasserbad nicht in die Platinverbindung des Neurins übergeführt werden kann. Ich habe zur Entscheidung dieser Frage einen $1 / 2$ Liter-Kolben mit $250 \mathrm{ccm} 0,5 \%$ iger wässeriger Cholinchloridlösung an drei verschiedenen Tagen im WasserdampfSterilisationsapparat ca. 15 Minuten auf $100^{\circ} \mathrm{C}$. erhitzt, dann die Flüssigkeit eingedampft bis zur Sirupdicke, wiederholt mit absolutem Alkohol extrahiert, den Alkohol verjagt und den Rückstand mit salzsäurehaltigem Wasser aufgelöst. Nach Zusatz von Platinchlorid krystallisierten, neben geringen Mengen der schwer löslichen, goldgelben, auf dem Platinblech dekrepitierenden Platin-

1) Arch. f. exp. Pharmakol., Bd. IV, 1875.

2) Ibidem, Bd. XX, 1886. 
salmiakkrystalle, beim weiteren freiwilligen Verdunsten nur die bekannten, rotgelben leichtlöslichen Tafeln aus, die häufig stufenweise übereinander lagen. Der Schmelzpunkt lag bei $234^{\circ} \mathrm{C}$, der Platingehalt betrug $31,57 \% \mathrm{Pt}$, während die Berechnung für Cholin $31,66 \% \mathrm{Pt}$ verlangt. Es scheint danach sicher, daß das Cholinchlorid eine dreimalige Erhitzung auf $100^{\circ} \mathrm{C}$. ohne irgendwelche Zersetzung, ganz besonders ohne eine Umwandlung in Neurin, erträgt.

Die eigentlichen Versuche gestalteten sich so, daß ich die saure Bouillon, bezw. das Peptonwasser ( $W$ it t e's Peton. sicc.) mit $0,5 \mathrm{~g}$ Cholinchlorid pro $100 \mathrm{ccm}$ Nährflüssigkeit versetzte, die Lösungen an drei aufeinanderfolgenden Tagen im Dampftopf sterilisierte, und dann von einer 24 stündigen Platte eine isolierte Kolonie impfte. Die Kolben wurden mit Gummikappen versehen.

\section{A. Versuche mit Oidlum lactis.}

Die drei Versuchsreihen, die ich im ganzen mit diesem gewöhnlichsten Schimmelpilz anstellte, unterscheiden sich in ihrer Versuchsanordnung nur insofern, als der erste nach vier Wochen, der zweite nach acht Wochen, der dritte nach drei Monaten unterbrochen wurde. Im Endeffekt gleichen sich alle drei, sodaß es sich erübrigt, nur einen und zwar den dritten etwas ausführlicher hier mitzuteilen. Am 2. September 1905 impfte ich fünf Kolben mit je $400 \mathrm{ccm}$ Cholinbouillon und überließ sie dem Wachstum bei Zimmertemperatur, die zwischen 18 und $20^{\circ} \mathrm{C}$. schwankte. Es bildete sich zunächst nach zwei Tagen im Zentrum der Flüssigkeit ein Pilzrasen, der schließlich die Oberfläche erreichte und dort zur Hautbildung führte. Allmählich sank dann dieser ganze Pilzrasen nach unten, die Hautbildung an der Oberfläche begann von neuem, und schließlich hatte sich eine große Masse gebildet, die am Volumen der Flüssigkeit nicht viel nachgab. Am 3. Dezember 1905 wurde der Versuch abgebrochen, nachdem makroskopisch kein Wachstum mehr zu verfolgen war. $\mathrm{Um}$ den Nachweis etwa gebildeter $\mathrm{CO}_{2}$ zu führen, hatte ich einen Kolben mit einer Gummikappe versehen, die in ihrer Mitte ein Ansatzstück zur Aufnahme einer Glasröhre trug. Diese Glasröhre war mehrmals rechtwinklig gebogen und führte $\mathrm{zu}$ einer $\mathrm{W}$ o u $\mathrm{l} f$ f'schen Waschflasche, mit der sie gasdicht verbunden war. Die Waschflasche selbst enthielt frisch bereitetes, klares Barytwasser und trug am anderen Ende ein AbsorptionsgefäB, das mit festem $\mathrm{NaOH}$ gefüllt war, um die Kohlensäure der Luft dort festzuhalten. Schon am zweiten Tage des Wachstums zeigte sich in der Waschflasche eine starke Trübung, die sich nach 
mehreren Tagen zu einem körnigen Niederschlag von $\mathrm{BaCO}_{3}$ verdichtete. Zeitweise konnte man die $\mathrm{CO}_{2}$ in Blasen einströmen sehen. Um sicher zu sein, daß die Kulturen während des Wachstums nicht durch andere Bakterien verunreinigt waren, habe ich von jedem der Kolben vor der chemischen Verarbeitung zwei Plattenserien mit je zwei Verdünnungen angelegt, von denen die eine bei ca. 18 bis $20^{\circ} \mathrm{C}$., die andere bei $37^{\circ} \mathrm{C}$. gehalten wurde. Nach vier Tagen zeigten die Platten im Brutschrank die ersten Kolonien von dem Oidium lactis, während sie bei Zimmertemperatur schon nach zwei Tagen reichlich keimten. Außer den charakteristischen Schimmelpilzkolonien mit ihrem sternförmigen Mycel wuchs auf den Platten nichts; eine Verunreinigung durch andere Mikroorganismen war somit auszuschließen.

Zur chemischen Verarbeitung wurde die Kulturflüssigkeit, die keinen auffallenden Geruch zeigte und gegen Lackmus schwach alkalisch reagierte, mit verdünnter Salzsäure schwach angesäuert, dann von dem Pilzrasen abfiltriert, auf dem Wasserbade eingedampft und so lange mit 96\% igem Alkohol ausgezogen, bis eine neue Probe nach dem Verdampfen des Alkohols keinen wesentlichen Rückstand mehr hinterließ. Die alkoholischen Auszüge wurden vereinigt, der Alkohol verdunstet und der sirupöse Rückstand mit HCl-haltigem Wasser aufgenommen. Trotzdem die wässerige Lösung nur sehr schwach gelblich gefärbt war, schied sich beim Zusatz von Platinchlorid, wie mich die ersten beiden Versuche mehrfach belehrt hatten, metallisches Platin als spiegelnde Haut aus, nachdem zuvor Platinsalmiak sich reichlich ausgeschieden hatte. Ich bin deshalb bei diesem Versuch so vorgegangen, daß ich fraktioniert mit $10 \%$ iger Goldchloridlösung gefällt habe, die jedesmalige Fällung mehrmals aus heißem Wasser umkrystallisiert und dann eine Schmelzpunkt- und eine Goldbestimmung ausgeführt habe. In der Regel genügte ein zweimaliges Umkrystallisieren um Goldsalze zu erhalten, die frei von reduziertem Gold waren. $\mathrm{Um}$ bei den Au-Bestimmungen kein Material zu verlieren, habe ich die abgewogene Menge in heißem Wasser gelöst, Schwefelwasserstoff eingeleitet, bis die über den Niederschlag befindliche Flüssigkeit absolut klar und ungefärbt war, dann das Schwefelgold auf einen quantitativen Filter gesammelt und wiederholt ausgewaschen. Filter und Niederschlag wurden bei $100^{\circ} \mathrm{im}$ Wassertrockenschrank getrocknet, dann in Pt-Spirale verascht und in einem gewogenen Porzellantiegel das Schwefelgold durch vorsichtiges Erhitzen zu Au reduziert, geglüht und bis zur Konstanz gewogen. 
1. Goldfällung. Nach dem Umkrystallisieren resultierten zunächst federbartartig angeordnete Nadeln, deren Schmelzpunkt bei $242^{\circ} \mathrm{C}$. liegt.

$0,2269 \mathrm{~g}$ getrocknete Substanz ergaben $0,1012 \mathrm{~g} \mathrm{Au}$; demnach gefunden $44,60 \% \mathrm{Au}$, berechnet für Cholin-Au-Salz $44,51 \%$.

Beim weiteren Verdunsten schieden sich neben den analysierten Krystallen kleine warzenförmige Krystalle aus, die durch Auslesen und Siebung getrennt werden. Der Schmelzpunkt dieser Krystalle lag bei $232^{\circ} \mathrm{C}$. Da ihre Menge zu gering zu einer Goldbestimmung war, so werden sie zunächst aufgehoben. Aus der Mutterlauge schieden sich weiterhin ziemlich große, unregelmäßig gestaltete, flitterartige Nadeln aus, deren Schmelzpunkt bei $240^{\circ} \mathrm{C}$. lag.

$0,1956 \mathrm{~g}$ Substanz ergaben $0,0875 \mathrm{~g} \mathrm{Au}$; demnach gefunden 44,73\% Au, berechnet für Cholin-Au-Salz 44,51\% Au.

2. Goldfällung. Federbartartige Krystalle. Schmelzpunkt $240^{\circ} \mathrm{C}$.

$0,2047 \mathrm{~g}$ Substanz ergaben $0,0916 \mathrm{~g} \mathrm{Au}$; demnach gefunden $44,75 \% \mathrm{Au}$, berechnet für Cholin-Au-Salz 44,51\% Au.

3. G o ld f äll u n g. Neben den typischen Cholingoldchloridkrystallen schieden sich, hauptsächlich an den Seitenwänden, punktförmige, warzige Krystalle aus, die nach dem Absaugen und Trocknen durch Stramin sich leicht voneinander trennen ließen.

$0,2122 \mathrm{~g}$ Substanz lieferten $0,0947 \mathrm{~g} \mathrm{Au}$; demnach gefunden 44,62\% Au, berechnet für Cholin-Au-Salz 44,51\% Au.

4. Goldfällung. Krystallform wie bei der 3.; auch hier traten wieder jene eigentümlichen kleinen, punktförmigen Krystallisationen auf, die auch hier wieder ausgelesen und getrennt wurden. Schmelzpunkt der federbartartigen Krystalle $238-239^{\circ} \mathrm{C}$.

$0,1870 \mathrm{~g}$ Substanz ergaben $0,0832 \mathrm{~g} \mathrm{Au}$; gefunden $44,49 \% \mathrm{Au}$, berechnet für Cholin-Au-Salz 44,51\%.

Die Mutterlaugen dieser ersten vier Fällungen wurden hierauf vereinigt und weiter der freiwilligen Verdunstung überlassen. Es schieden sich hierbei weiter große, zum Teil flitterförmige, zum Teil mehr tafelförmige Krystalle aus, deren Schmelzpunkt bei $240^{\circ} \mathrm{C}$. lag.

$0,3287 \mathrm{~g}$ Substanz ergaben 0,1084 g Gold; demnach gefunden 44,78\% Au, berechnet für Cholin-Au 44,51\%.

Trotz ihres wesentlich verschiedenen Aussehens dürfte es sich bei letzteren Krystallen doch auch nur um Cholingoldchlorid gehandelt haben. Aus den letzten Resten dieser vereinigten Mutter- 
laugen schieden sich noch größere, kompaktere, tafelförmige Krystalle aus, die äußerst leicht löslich waren. Sie wurden gesammelt, in Wasser gelöst, mit $\mathrm{H}_{2} \mathrm{~S}$ entgoldet und in möglichst ammoniakfreier Atmosphäre mit Platinchlorid versetzt. Es krystallisierten alsdann sehr schwer lösliche, reguläre Oktaeder aus, die auf dem Pt-Blech dekrepitierten. Da aus der ersten Mutterlauge sich inzwischen viel metallisches Gold ausgeschieden hatte, so wurde die Lösung ebenfalls mit $\mathrm{H}_{2} \mathrm{~S}$ entgoldet; es restierte hierdurch eine gelbe Flüssigkeit, die zur weiteren Klärung mit 2-3 Tropfen konzentrierter wässeriger Sublimatlösung versetzt wurde. Eine Fällung wurde dabei nicht beobachtet. Nach erneutem Einleiten von Schwefelwasserstoff, zwecks Ausfällung des Quecksilbers, restierte eine klare, gelbe Flüssigkeit, die nun weiterhin mit Goldchloridlösung gefällt wurde.

5. G o l d f äll u n g. Punktförmige, warzige Krystalle fallen ohne Beimengungen anderer Krystallformen aus. Diese werden mit den gleichartigen Produkten der ersten Fällung vereinigt und umkrystallisiert. Schmp. $239^{\circ} \mathrm{C}$.

$0,1596 \mathrm{~g}$ Substanz ergaben $0,0713 \mathrm{~g} \mathrm{Au}$; gefunden $44,67 \% \mathrm{Au}$, berechnet für Cholin-Au-Salz 44,51\% Au.

Beim weiteren Verdunsten der Mutterlauge dieser Krystallisation zeigten sich federbartartige Krystalle, deren Schmelzpunkt bei $241^{\circ} \mathrm{C}$. lag.

$0,2458 \mathrm{~g}$ Substanz lieferten $0,1094 \mathrm{~g} \mathrm{Au}$; gefunden $44,51 \% \mathrm{Au}$, berechnet für Cholin-Au-Salz 44,51\% Au.

6. Go ld f äll u n g. Nadelförmige Krystalle, deren Schmelzpunkt bei $244^{\circ} \mathrm{C}$. lag.

$0,2126 \mathrm{~g}$ Substanz lieferten $0,0949 \mathrm{~g} \mathrm{Au}$; gefunden 44,64\% $\mathrm{Au}$, berechnet für Cholin-Au-Salz 44,51\% Au.

7. Gold f äll u ng. Zunächst punktförmige Krystalle vom Schmp. $240^{\circ} \mathrm{C}$, dann tafelförmige, große, unregelmäßig gestaltete Flittern vom Schmp. $242^{\circ} \mathrm{C}$. Von den letzteren Krystallen lieferten

$0,2011 \mathrm{~g}$ Substanz $0,0897 \mathrm{~g} \mathrm{Au}$; also gefunden $44,60 \% \mathrm{Au}$, berechnet für Cholin-Au-Salz 44,51\% Au.

8. Goldfällu ng. Neben wenigen warzenförmigen Krystallen, kompaktere federbartartige, cholinähnliche vom Schmp. $243^{\circ} \mathrm{C}$.

$0,2237 \mathrm{~g}$ Substanz lieferten $0,0991 \mathrm{~g} \mathrm{Au}$; gefunden $44,30 \% \mathrm{Au}$, berechnet für Cholin-Au-Salz 44,51\% Au.

9. Gold f äll u n g. Nadelförmige und federbartartige Krystalle vom Schmp. $244^{\circ} \mathrm{C}$. 
$0,2126 \mathrm{~g}$ Substanz lieferten $0,0949 \mathrm{~g} \mathrm{Au}$; gefunden $44,64 \% \mathrm{Au}$, berechnet für Cholin-Au-Salz 44,51\% Au.

10. G o ldfällu ng. Nadelförmige Krystalle vom Schmelzpunkt $241^{\circ} \mathrm{C}$.

$0,2116 \mathrm{~g}$ Substanz lieferten $0,0950 \mathrm{~g} \mathrm{Au}$; gefunden $44,80 \% \mathrm{Au}$, berechnet für Cholin-Au-Salz 44,51\% Au.

Die Mutterlaugen von dieser 5. bis 10. Goldfällung wurden vereinigt, zur Reinigung von dem ausgeschiedenen metallischen Gold etc. mit $\mathrm{H}_{2} \mathrm{~S}$ entgoldet, das Filtrat eingedampft und erneut mit Goldchlorid versetzt. Es schieden sich bei freiwilliger Verdunstung nur nadelförmige Krystalle vom Schmp. $240^{\circ} \mathrm{C}$. aus.

$0,2691 \mathrm{~g}$ Substanz lieferten $0,1204 \mathrm{~g} \mathrm{Au}$; gefunden $44,73 \% \mathrm{Au}$, berechnet für Cholin-Au-Salz 44,51\% Au.

Die restierenden Mengen wurden mit $\mathrm{H}_{2} \mathrm{~S}$ entgoldet, der $\mathrm{H}_{2} \mathrm{~S}$ bei mäßiger Wärme verjagt, und das Filtrat mit den übrigen Filtraten aller Au-Fällungen vereinigt, und mit Platinchlorid versetzt. Ueber $\mathrm{H}_{2} \mathrm{SO}_{4}$ im Exsikkator krystallisierten zunächst goldgelbe, schwer lösliche Oktaeder aus, die getrocknet, auf dem Pt-Blech dekrepitierten. Da ihre Menge nicht groß war, so wurden die Krystalle gesammelt und im Exsikkator aufbewahrt. Bei weiterer Verdunstung fielen noch tafelförmige, rotgelbe, zum Teil sehr große (bis $1 \mathrm{~cm}$ im größten Durchmesser) Krystalle aus, die nach ihrem Schmp. $235^{\circ} \mathrm{C}$. und ihrem Pt-Gehalt, sowie nach ihrer leichten Löslichkeit mit Cholin-Pt-Chlorid identisch waren.

$0,2135 \mathrm{~g}$ Substanz lieferten $0,0678 \mathrm{~g} \mathrm{Pt}$; gefunden $31,38 \% \mathrm{Pt}$, berechnet für Cholinplatinchlorid $31,66 \% \mathrm{Pt}$.

Es sei hier ausdrücklich betont, daß gerade bei den Pt-Krystallisationen auf die schwerlöslichen Anteile besonders geachtet wurde, da hier das eventuell gebildete Neurinplatinchlorid sich hätte besonđers leicht zeigen müssen. Die primäre Mutterlauge hatte nach Entfernung des Goldchlorids durch Schwefelwasserstoff eine solche Farblosigkeit erreicht, daß der Versuch gerechtfertigt schien, die darin enthaltenen Basen weiterhin mit Hilfe von Platinchlorid zu isolieren. Der Niederschlag von Schwefelgold wurde ca. 20 mal mit warmen, HCl-haltigem Wasser ausgewaschen, dann das Filtrat eingeengt und mit Platinchlorid versetzt. Es schieden sich zunächst im Exsikkator über $\mathrm{H}_{2} \mathrm{SO}_{4}$ große Mengen von goldgelben Oktaedern aus, von denen eine Probe auf dem Pt-Blech durch Dekrepitieren sich als anorganisch erwies. Diese Krystalle wurden mit den oben genannten, aus den ersten 10 Krystallisationen gewonnen, vereinigt, umkrystallisiert und gesammelt. 
$0,2156 \mathrm{~g}$ Substanz lieferten $0,9472 \mathrm{~g} \mathrm{Pt}$; gefunden $43,94^{\circ}{ }_{\omega} \mathrm{Pt}$, berechnet für $\left(\mathrm{NH}_{4}\right)_{2} \mathrm{PtCl}_{6} 43,91 \% \mathrm{Pt}$.

Die Mutterlauge zeigte beim weiteren Verdunsten nur geringe Neigung zur Krystallisation, sie wurde deshalb mit absolutem Alkohol gefällt, bis nach Sedimentierung des gelblichen Niederschlages weiterer Alkoholzusatz keine Fällung mehr hervorrief. Aus dem getrockneten, in HCl-haltigem Wasser gelösten Niederschlag der Alkoholfällung kamen zunächst reichlich große tafelförmige, braunrote Krystalle heraus, die in ihrem Aussehen ganz wie Cholin-Pt-Salz aussahen. Schmp. $236^{\circ} \mathrm{C}$. Außerdem schieden sich noch gelbe, eigentümliche, matt aussehende, tropfenförmige Gebilde aus, die ausgelesen und nach Zusatz einiger Tropfen Platinchloridlösung umkrystallisiert wurden. Ueber $\mathrm{H}_{2} \mathrm{SO}_{4}$ verdunstet, schieden sich daraus goldgelbe oktaedrische Krystalle von Platinsalmiak und typische Tafeln von Cholinplatinchlorid aus, deren Schmelzpunkt bei $235^{\circ} \mathrm{C}$. lag. Es hatte sich also scheinbar bei jenen tropfenförmigen Gebilden um eine Vergesellschaftung von Cholin- und Ammoniumplatinchlorid gehandelt. Die Mutterlauge von dieser ersten Alkoholfällung wurde zwecks Einengung des Volumens der freiwilligen Verdunstung überlassen, und dann mit alkoholischer Platinchloridlösung weiter gefällt. Auf diese Weise crhielt ich noch zwei größere Pt-Fällungen, die vollkommen getrocknet und aus HCl-haltigem Wasser umkrystallisiert wurden. Es fielen aus beiden Fällungen nur tafelförmige, gelbrote, leicht lösliche Krystalle aus. Schmelzpunktbestimmungen ergaben für die erste alkoholische Fällung $234^{\circ} \mathrm{C}$, für die zweite $235-236^{\circ} \mathrm{C}$. Die Platinbestimmung ergab für die erste Alkoholfällung: Pt-Gehalt.

$0,1943 \mathrm{~g}$ Substanz ergaben $0,0618 \mathrm{~g} \mathrm{Pt}$; entsprechend $31,85^{\circ}$, für die zweite alkoholische Fällung:

$0,2354 \mathrm{~g}$ Substanz ergaben $0,0747 \mathrm{~g} \mathrm{Pt}$; gefunden $31,76^{\circ} \mathrm{o} \mathrm{Pt}$, berechnet für Cholinplatinchlorid $31,66 \% \mathrm{Pt}$.

Die alkoholische Mutterlauge zeigte bei Zusatz von Platinchlorid (alkoholisch) keine weiteren Fällungen noch Krystallisationen, es wurde deshalb nach freiwilliger Verdunstung des Alkohols durch Einleiten von $\mathrm{H}_{2} \mathrm{~S}$ das Platin successive ausgefällt, der Schwefelplatinniederschlag reichlich ausgewaschen und das Filtrat eingeengt. Da eine kleine Probe mit Wismutjodidjodkali in schwefelsaurer Lösung einen bräunlichroten, ölig-beweglichen, nicht krystallisierten Niederschlag gab, so wurde das ganze Filtrat mit Wismutjodidjodkaliumlösung gefällt, der Niederschlag gesammelt, 
in Wasser suspendiert, und dann mit $\mathrm{H}_{2} \mathrm{~S}$ das Wismut und mit frisch bereitetem Chlorsilber das Jod entfernt. Das eingeengte Filtrat wurde mit wässeriger Platinchloridlösung versetzt; es krystallisierten bei langsamer Verdunstung feine lange Nadeln und kleine warzige Krystalle von braunroter Farbe aus, die ziemlich leicht löslich waren. Die Menge der Krystallisationen war so gering, daß von einer exakten chemischen Untersuchung Abstand genommen wurde. Vielmehr habe ich damit physiologische Versuche an Meerschweinchen und Fröschen angestellt. $\mathrm{Zu}$ diesem Zwecke habe ich diese Krystallisationen gesammelt, den Rest der darin enthaltenen Basen mit Alkohol, absolut. und Zusatz von Pt-Chlorid ausgefällt, über $\mathrm{H}_{2} \mathrm{SO}_{4}$ im Exsikkator getrocknet, dann gepulvert und bis zur Konstanz gewogen. Ich erhielt auf diese Weise noch $0,2035 \mathrm{~g}$ Pt-Salz, das dann durch $\mathrm{H}_{2} \mathrm{~S}$ quantitativ in das Chlorid ( $=0,0922 \mathrm{~g}$ für Cholin berechnet) übergeführt wurde.

Nach Verjagen des $\mathrm{H}_{2} \mathrm{~S}$ und vorsichtigem Eindampfen wurde der Rückstand in Wasser gelöst.

Von den Versuchen seien folgende erwähnt:

Große Esculenta von $78 \mathrm{~g}$ Gewicht. Herz freigelegt.

$11 \mathrm{Uhr} 45$ Min. . . . 16 Kontraktionen in 20 Sekunden

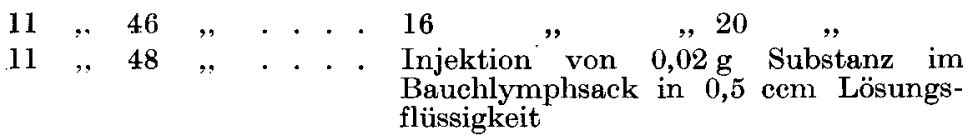

$11,50, \%$. . . . 16 Kontraktionen in 20 Sekunden

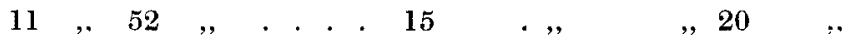

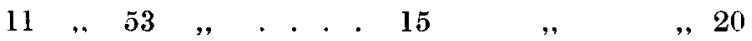

$11,, 54 \quad, \quad . . .615$

$11,, 55 \quad, \quad . \quad . \quad .14$

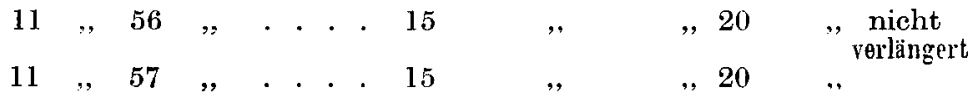

$\quad ", \quad, 20 \quad, 20$

$12,00,0 . \ldots . .15$

$12, \quad 05 \quad, \quad . \quad . \quad . \quad 15$

$12, " 08, \% \quad . \quad . \quad . \quad 15$

$12,, 10, \%$. . . . 15

$12,, 20, \ldots, \ldots . \quad .15$

$12, \quad 30, \ldots, \ldots . \quad 15$

$1 \quad, \quad 00 \quad, \quad . . . .015$

Versuch abgebrochen. 
5 Uhr läuft im Käfig herum, frißt reichlich.

6 Uhr vollkommen munter.

11. August 1906. Morgens.

Bietet nichts Auffälliges.

Es sei hier erwähnt, daß das Filtrat von der Wismutjodidjodkaliumfällung mit $\mathrm{H}_{2} \mathrm{~S}$ vom Wismut und dann mit $\mathrm{AgCl}$ vom Jod befreit wurde. Diese letzte Mutterlauge wurde weiter mit den verschiedensten Alkaloidfällungsmitteln (Phosphorwolframsäure, Jodjodkali etc.) versetzt, ohne daß ein Resultat erzielt werden konnte. Man darf danach wohl annehmen, daß wesentliche Mengen von Basen nicht mehr darin waren.

Mit der fraktionierten Fällung, wie sie oben beschrieben ist, habe ich im ganzen ca. 14,5 g Platindoppelsalz des Cholins wieder gewonnen, die auf Cholinchlorid berechnet, eine Menge von $6,5 \mathrm{~g}$ darstellen würden. Es waren mithin 3,5 $\mathrm{g}$ Cholinchlorid in den Kulturen von den Schimmelpilzen verbraucht worden.

\section{B. Versuche mit Vibrio cholerae.}

Von den zwei Versuchen, die ich mit dem Vibrio cholerae angestellt habe, will ich ausführlicher den zweiten mitteilen, der sich in der Versuchsanordnung nur dadurch vom ersten unterscheidet, daß die Wachstumszeit zwei Monate betrug, während sich beim ersten diese Zeit nur auf einen Monat belief. Am 6. August 1905 impfte ich von einer 24 stündigen Gelatinekolonie fünf Kolben mit je $400 \mathrm{ccm} \mathrm{0,5 \%} \mathrm{Cholinchlorid-Peptonwasser} \mathrm{und} \mathrm{ließ} \mathrm{dieselben}$ bei $37 \mathrm{C}$. im Brutschrank wachsen. Schon am nächsten Tage zeigte sich eine starke Trübung der ganzen Flüssigkeit, die sich allmählich zu einem gelben reichlichen Bodensatz verdichtete. Häutchenbildung ließ sich dabei nicht beobachten. Einen dieser Kolben hatte ich ebenso wie oben bei dem Oidium-lactis-Versuch, gasdicht mit einem $\mathrm{Ba}(\mathrm{OH})_{2}$-Gefäß verbunden. Es zeigte sich schon am zweiten Tage eine reichliche Trübung und Abscheidung ven $\mathrm{BaCO}_{3}$. Am 6. Oktober 1905 habe ich das Wachstum unterbrochen und zur Kontrolle auf Reinheit je drei Gelatineplatten mit zweifacher Verdünnung gegossen. Außer den charakteristischen Cholerakolonien wuchs nichts auf demselben. An Präparaten im hängenden Tropfen, sowie an gefärbten Präparaten fiel der große Reichtum an Spirillenformen auf.

Die chemische Verarbeitung gestaltete sich in diesem Versuch zunächst so, daß ich die stark alkalisch reagierende Flüssigkeit unter dauernder Luftdurchleitung bei einer Temperatur, die $50^{\circ} \mathrm{C}$. nicht überstieg, destillierte, um eventuell flüchtige Basen über- 
zutreiben. Das Destillat wurde in einer Vorlage mit $\mathrm{HCl}$-haltigem Wasser aufgefangen. Auf diese Weise habe ich fünf Stunden destilliert, das Destillat eingedampft, wobei sich am Rand ein weißliches Krystallpulver abschied. Beim Zusatz von Pt-Chloridlösung schieden sich massenhaft goldgelbe, schwer lösliche Oktaeder ab, die nicht schmolzen und bei der Pt-Bestimmung sich als Platinsalmiak charakterisierten.

$0,2351 \mathrm{~g}$ Substanz lieferten $0,1034 \mathrm{~g}$ Pt; demnach gefunden $43,98 \% \mathrm{Pt}$, berechnet für $\left(\mathrm{NH}_{4}\right)_{2} \mathrm{PtCl}_{6} 43,91 \% \mathrm{Pt}$.

Um sicher zu sein, daß nicht in den Mutterlaugen dieser Ausscheidung, noch irgendwelche andere, leicht lösliche Anteile versteckt blieben, habe ich dieselben mit $\mathrm{H}_{2} \mathrm{~S}$ vom $\mathrm{Pt}$ befreit, das Filtrat eingeengt und mit Goldchlorid versetzt. Erst nach längerem Verdunsten schieden sich große, goldgelbe, äußerst leicht lösliche Tafeln aus, die nicht schmolzen und nach dem Goldgehalt ebenfalls Goldsalmiak waren.

$0,2756 \mathrm{~g}$ Substanz (bei $100^{\circ}$ getrocknet) lieferten $0,1512 \mathrm{~g} \mathrm{Au}$; gefunden $52,95 \% \mathrm{Au}$, berechnet für $\left(\mathrm{NH}_{4}\right) \mathrm{AuCl}_{4}+1 \mathrm{H}_{2} \mathrm{O}: 52,46 \% \mathrm{Au}$.

Nach beendeter Destillation habe ich die Kulturflüssigkeit mit $\mathrm{HCl}$ schwach angesäuert, eingedampft, den Rückstand mit Alkohol erschöpft, von den alkoholischen Auszügen den Alkohol verjagt, den Rückstand mit Wasser aufgenommen und fraktioniert mit Goldchlorid gefällt. Ich habe auf diese Weise 17 Goldfällungen gewonnen.

1. Goldfällu ng. Federbartartig angeordnete, schwer lösliche Krystalle vom Schmp. $239^{\circ} \mathrm{C}$.

$0,2283 \mathrm{~g}$ Substanz liefern $0,1020 \mathrm{~g} \mathrm{Au}$; demnach gefunden $44,67 \% \mathrm{Au}$, berechnet für Cholin-Goldsalz $44,51 \%$ \%

2. Goldfällung. Krystallform zum Teil feine Nadeln, zum Teil federbartartig angeordnet. Schmp. $243^{\circ} \mathrm{C}$.

$0,2202 \mathrm{~g}$ Substanz liefern $0,0988 \mathrm{~g} \mathrm{Au}$; demnach gefunden $44,85 \% \mathrm{Au}$, berechnet für Cholin-Goldsalz $44,51 \%$.

3. Goldfällung. Krystallform nadelförmig, Schmelzpunkt $243^{\circ} \mathrm{C}$.

$0,2226 \mathrm{~g}$ Substanz liefern $0,098 \pm \mathrm{g} \mathrm{Au}$; demnach gefunden $44,21 \% \mathrm{Au}$, berechnet für Cholin-Goldsalz 44,51\%.

4. Goldfällu ng. Krystallform federbartartig, Schmelzpunkt $241^{\circ} \mathrm{C}$.

$0,2190 \mathrm{~g}$ Substanz liefern $0,0975 \mathrm{~g} \mathrm{Au}$; demnach gefunden $44,52 \% \mathrm{Au}$, berechnet für Cholin-Goldsalz 44,51\% $\%$.

5. Goldfällu ng. Krystallform wie bei der 4. Fällung. Schmp. $240^{\circ} \mathrm{C}$. 
$0,2218 \mathrm{~g}$ Substanz liefern $0,0990 \mathrm{~g} \mathrm{Au}$; demnach gefunden $44,62 \% \mathrm{Au}$, berechnet für Cholin-Goldsalz 44,51\%.

6. Goldfällung. Krystallform federbartartig angeordnete Nadeln. Schmp. $243^{\circ} \mathrm{C}$.

$0,2208 \mathrm{~g}$ Substanz liefern $0,1010 \mathrm{~g} \mathrm{Au}$; demnach gefunden $44,53 \% \mathrm{Au}$, berechnet für Cholin $44,51 \%$.

7. Goldfällung. Krystallform ohne Besonderheiten. Schmp. $241^{\circ} \mathrm{C}$.

$0,2218 \mathrm{~g}$ Substanz liefern $0,0990 \mathrm{~g} \mathrm{Au}$; demnach gefunden $44,63 \% \mathrm{Au}$, berechnet für Cholin $44,51 \% \mathrm{Au}$.

8. Goldf äll u ng. Krystallform nadelförmig. Schmelzpunkt $243^{\circ} \mathrm{C}$.

$0,2114 \mathrm{~g}$ Substanz liefern $0,0946 \mathrm{~g} \mathrm{Au}$; demnach gefunden $44,75 \% \mathrm{Au}$, berechnet für Cholin $44,51 \% \mathrm{Au}$.

9. Gold fäll u ng. Krystallform federbartartig. Schmelzpunkt $244^{\circ} \mathrm{C}$.

$0,1768 \mathrm{~g}$ Substanz liefern $0,0785 \mathrm{~g} \mathrm{Au}$; demnach gefunden $44,40 \% \mathrm{Au}$, berechnet für Cholin $44,51 \% \mathrm{Au}$.

10. Go ld fällu ng. Krystallform nadelförmig. Schmelzpunkt $243^{\circ} \mathrm{C}$.

$0,1889 \mathrm{~g}$ Substanz liefern $0,0842 \mathrm{~g} . \mathrm{Au}$; demnach gefunden $44,57 \% \mathrm{Au}$, berechnet für Cholin $44,51 \% \mathrm{Au}$.

11. Goldfällung. Krystallform federbartartig mit Nadeln. Schmp. $241^{\circ} \mathrm{C}$.

$0,2129 \mathrm{~g}$ Substanz liefern $0,0952 \mathrm{~g} \mathrm{Au}$; demnach gefunden $44,71 \% \mathrm{Au}$, berechnet für Cholin $44,51 \% \mathrm{Au}$.

12. Go ld f äll u n g. Krystallform nadelförmig. Schmelzpunkt $240^{\circ} \mathrm{C}$.

$0,2631 \mathrm{~g}$ Substanz liefern $0,1171 \mathrm{~g} \mathrm{Au}$; demnach gefunden $44,51 \% \mathrm{Au}$, berechnet für Cholin $44,51 \% \mathrm{Au}$.

13. Gold fäl lu $\mathrm{n}$ g. Krystallform federbartartig. Schmelzpunkt $241^{\circ} \mathrm{C}$.

$0,2327 \mathrm{~g}$ Substanz liefern $\mathbf{0 , 1 0 3 8} \mathrm{g} \mathrm{Au}$; demnach gefunden $\mathbf{4 4 , 6 0 \% ~ A u , ~ b e r e c h n e t ~ f u ̈ r ~ C h o l i n ~} 44,51 \% \mathrm{Au}$.

14. Goldfällung. Krystallform wie bei Fällung 11 . Schmp. $242^{\circ} \mathrm{C}$.

$0,1875 \mathrm{~g}$ Substanz liefern $0,0834 \mathrm{~g} \mathrm{Au}$; demnach gefunden $44,48 \% \mathrm{Au}$, berechnet für Cholin $44,51 \% \mathrm{Au}$.

15. G oldfällung. Krystallform meist lange Nadeln. Schmp. $240^{\circ} \mathrm{C}$.

$0,2372 \mathrm{~g}$ Substanz liefern $0,1060 \mathrm{~g} \mathrm{Au}$; demnach gefunden $44,68 \% \mathrm{Au}$, berechnet für Cholin $44,51 \% \mathrm{Au}$. 
16. Goldfällung. Krystallform federbartartig mit Nadeln. Schmp. $243^{\circ} \mathrm{C}$.

$0,2348 \mathrm{~g}$ Substanz liefern $0,1038 \mathrm{~g} A \mathrm{Au}$; demnach gefunden $44,21 \% \mathrm{Au}$, berechnet für Cholin $44,51 \% \mathrm{Au}$.

17. Gold f äll u ng. Krystallform nadelförmig. Schmelzpunkt $240^{\circ} \mathrm{C}$.

$0,2541 \mathrm{~g}$ Substanz liefern $0,1135 \mathrm{~g} \mathrm{Au}$; demnach gefunden $44,66 \%$ Au, berechnet für Cholin $44,51 \% \mathrm{Au}$.

Da diese sämtlichen Analysen analog den Versuchen mit Oidium lactis unter Anwendung von $\mathrm{H}_{2} \mathrm{~S}-\mathrm{F}$ ällung ausgeführt waren, so wurden die Filtrate von jeder Analyse gesammelt und das Pt-Salz übergeführt. Die restierenden Mutterlaugen der einzelnen Au-Fällungen wurden mit $\mathrm{H}_{2} \mathrm{~S}$ entgoldet und gleichfalls in die Platinverbindungen übergeführt. Neben goldgelben, oktaedrischen schwer löslichen Krystallen von Platinsalmiak, schieden sich aus beiden Krystallisationen nur tafelförmige, relativ leicht lösliche Krystalle aus, deren Schmelzpunkt bei $234-236^{\circ} \mathrm{C}$. lag, während die ersteren auf dem Platinblech dekrepitierten. Neurinähnliche Krystallisationen wurden unter den schwer löslichen Anteilen der Platinsalze nicht beobachtet. Die letzten Reste dieser Platinverbindungen wurden durch Alkohol abgeschieden, und zwar solange, bis ein weiterer Zusatz von alkoholischem Platinchlorid keinen Niederschlag mehr gab. Von zwei Stichproben dieser Platinverbindungen wurden $\mathrm{Pt}$-Bestimmungen ausgeführt:

1. 0,2058 g Pt-Salz ergaben $0,0647 \mathrm{~g} \mathrm{Pt}$; demnach gefunden $31,52 \% \mathrm{Pt}$, berechnet für Cholin-Pt-Salz $31,66 \% \mathrm{Pt}$.

2. $0,2478 \mathrm{~g} \mathrm{Pt-Salz} \mathrm{liefern} 0,0778 \mathrm{~g} \mathrm{Pt}$; demnach gefunden $31,43 \% \mathrm{Pt}$, berechnet für Cholin-Pt-Salz 31,66\% Pt.

Die Gesamtmenge der Cholin-Platinsalze betrug etwa 12,3 g, einer Menge, der 5,5 g Cholinchlorid äquivalent sein dürfte. Die Mutterlauge, die von der 17. Goldfällung zurückblieb, zeigte auch im Exsikkator keine Neigung mehr zur Krystallisation. Sie wurde deshalb mit $\mathrm{H}_{2} \mathrm{~S}$ entgoldet und mit Platinchlorid versetzt. Hierbei schieden sich nach längerem Verdunsten ganz helle, durchsichtige, quadratische Tafeln aus, die sich als $\mathrm{NaCl}$ erwiesen. Es wurde nun mit ablosutem Alkoholäther gefällt, wobei sich noch ein scheinbar reichlicher Niederschlag bildete. Derselbe wurde gesammelt, vollkommen getrocknet und in Wasser gelöst. Es schieden sich zunächst feine Nadeln aus, die leicht löslich waren, aber nach mehrmaligem Umkrystallisieren Tafelform annahmen. Der Schmelzpunkt lag bei $232^{\circ} \mathrm{C}$. 
$0,1756 \mathrm{~g}$ Substanz lieferten $0,0552 \mathrm{~g}$ Platin; demnach gefunden $31,75 \% \mathrm{Pt}$, berechnet für Cholin-Pt-Salz $31,66 \% \mathrm{Pt}$.

Außer diesen nadelförmigen bezw. tafelförmigen Krystallen schieden sich schließlich noch kleine rötliche, warzenförmige Krystalle aus, allerdings in so geringer Menge, daß sie zu einer chemischen Verarbeitung nicht genügten. Sie wurden gesammelt und zu einer später zu besprechenden physiologischen Prüfung verwandt. Das Filtrat der oben genannten Alkoholätherfällung wurde der Verdunstung überlassen, ohne daß etwas auskrystallisierte. Daraufhin wurde das Platin mit $\mathrm{H}_{2} \mathrm{~S}$ ausgefällt, der $\mathrm{H}_{2} \mathrm{~S}$ verjagt und das Filtrat nach Ansäuerung mit $\mathrm{H}_{2} \mathrm{SO}_{4}$ tropfenweise mit Wismutjodidjodkaliumlösung versetzt. Es fiel zunächst ein geringer, bräunlicher, öliger Niederschlag aus, der abfiltriert wurde, da ein weiterer Zusatz von $\mathrm{H}_{2} \mathrm{SO}_{4}$ und Wismutjodidjodkalium keine weitere Fällung mehr brachte. Eine Probe des Filtrates wurde versuchsweise mit Jodjodkaliumlösung versetzt, wobei sich ein körniger, roter Niederschlag bildete. Nachdem das ganze Filtrat mit dem gleichen Reagens ausgefällt war, wurden sämtliche Niederschläge vereinigt, mit wenig Alkohol verrieben und mit kohlensaurem Silber zerlegt, welch letzteres so lange zugesetzt wurde, bis ein Tropfen Goldehloridlösung keine braune Färbung mehr hervorrief. Das Gemenge wurde dann in salzsäurehaltigem Wasser ausgezogen, die Auszüge eingeengt und mit Platinchlorid versetzt. Beim Verdunsten im Exsikkator über Schwefelsäure schieden sich neben feinen, leicht löslichen rotbraunen Nadeln späterhin noch dunklere punktförmige Krystalle aus. Der Schmelzpunkt der Nadeln lag bei $230^{\circ} \mathrm{C}$. Die gewonnene Menge dieser Platinsalze war so gering, daß sie zu einer Platinbestimmung nicht mehr genügte. Sie wurde deshalb mit der oben erwähnten vereinigt und zur physiologischen Prüfung verwandt.

An Fröschen, deren Herz frei gelegt war, riefen Mengen von 5-10 mg des Chlorids der erwähnten Platinverbindungen keine sichtbaren Veränderungen der Kontraktionen hervor, ebenso wirkungslos waren auch Dosen von $20-50 \mathrm{mg}$ an Meerschweinchen und Katzen bei subkutaner Einverleibung. Die Herztätigkeit und Respiration blieben vollkommen unbeeinflußt.

\section{Zusammenfassung.}

Die vorstehenden Untersuchungen haben als positives Resultat ergeben, daß sowohl Oidium lactis, wie der Vibrio cholerae das Cholin in Kohlensäureanhydrid, Ammoniak und W a s s e r spalten. Dabei scheint die Zerlegung des Moleküls so 
vor sich zu gehen, daß direkt diese Endprodukte gebildet werden, denn $Z$ wischenprodukte, wie z. B. Trimethylamin, habe ich in keiner einzigen der Kulturen nachweisen können.

Das Wachstum beider Mikroorganismen war auf den cholinhaltigen Nährböden als ein sehr gutes zu bezeichnen. Von dem Cholera-Vibrio war es a priori wohl zu erwarten, da er ja bekanntermaßen besonders gut auf Eiern wächst, die ja reich an Lecithin, der Muttersubstanz des Cholins im tierischen Haushalt, sind. Erwähnenswert scheinen mir noch die Tatsachen, daß der CholeraVibrio bei seinem Wachstum auf diesen Cholinnährböden so auffallend viel Spirillen bildet.

Die weitere, sehr wichtige Frage, ob diese beiden Lebewesen in Reinkultur aus Cholin durch Wasserabspaltung $N$ e $u$ r in bilden können, müssen wir nach unseren Untersuchungen v e rn e i n e n, da wir trotz eifrigen Suchens, besonders auch in den reichlichen Salmiakprodukten, niemals die leicht zu erkennenden Neurinplatinchloridkrystalle gefunden haben. Daß auch in den letzten Mutterlaugen kein Neurin oder eine andere giftige Base vorhanden war, dürften die bezüglichen physiologischen Experimente beweisen.

Diese Frage hat mit Bezug auf den weit verbreiteten Schimmelpilz Oidium lactis insofern ein weittragendes praktisches Interesse, als ein großer Teil unserer Nahrungsmittel das Cholin gelegentlich frei als solches und ganz besonders gebunden in Form von L e cithin enthält. Was fernerhin den Cholera Vibrio angeht, so wäre ein Nachweis von Neurin selbstverständlich eine wichtige Erkenntnis für die Pathologie dieser Infektionskrankheit gewesen, zumal wir ja auch heute noch nichts Positives über das Choleragift trotz vielfacher Bemühungen wissen.

Wenn die Untersuchungen bezüglich der Bildung von Neurin negativ verlaufen sind, so dürfen sie doch insofern ein gewisses Interesse beanspruchen, als weitere Untersuchungsreihen mit anderen Mikroorganismen pathogener oder nicht pathogener Art neue Tatsachen zu Tage fördern können. Ganz besonders glaube ich, daß eventuell Versuche unter Symbiose, z. B. von Diphtheriebazillen und Streptokokken, für die Erkenntnis des Stoffwechsels dieser Organismen aussichtsvoll wären. Ein Beweis dafür liegt ja in den oben schon erwähnten Untersuchungen $E$. S c h m id t's, den ja der Nachweis von Neurin in Heuinfuskulturen gelang.

Herrn Geheimrat E. Sch midt in Marburg sei an dieser Stelle für die Anregung zu dieser Arbeit und das dauernde Interesse an derselben herzlichst gedankt. 